\title{
Barriers to Patient-physician Communication About Out-of-pocket Costs
}

\author{
G. Caleb Alexander, MD, MS, Lawrence P. Casalino, MD, PhD, Chien-Wen Tseng, MD, MPH, \\ Diane McFadden, BA, David O. Meltzer, MD, PhD
}

BACKGROUND: Though many patients and physicians believe that they should discuss out-of-pocket costs, research suggests that they infrequently do.

OBJECTIVE: To examine barriers preventing patient-physician communication about out-of-pocket costs among study subjects recalling a time when they wanted to discuss these costs but did not do so.

DESIGN, SETTING, AND PARTICIPANTS: Cross-sectional surveys of 133 general internists and 484 of their patients from 3 academic and 18 community practices in a large midwestern metropolitan region.

MEASUREMENTS: Patient- and physician-reported barriers to discussing out-of-pocket costs.

MAIN RESULTS: Overall, 54 patients (11\%) and 27 physicians $(20 \%)$ were able to recall a specific time when they wanted to discuss out-of-pocket costs but did not do so. Among patients, a wide variety of barriers were reported including their own discomfort (19\%), insufficient time (13\%), a belief that their physician did not have a viable solution (11\%), and concerns about the impact of discussions on quality of care (9\%). Among physicians, the most common barriers reported were insufficient time (67\%) and a belief that they did not have a solution to offer (19\%).

CONCLUSIONS: Efforts to promote discussions of out-of-pocket costs should emphasize the legitimacy of patients' concerns and brief actionable alternatives that physicians can take to address them.

KEY WORDS: patient-physician communication; out-of-pocket costs.

J GEN INTERN MED 2004;19:856-860.

A bout $20 \%$ of U.S. health care costs are paid by patients out-of-pocket. ${ }^{1}$ During the past 3 decades these costs have grown more than 8-fold from \$25.1 billion in 1970 to more than $\$ 205$ billion in $2001 .^{2}$ High

Received from the Robert Wood Johnson Clinical Scholars Program (GCA, LPC, DOM), MacLean Center for Clinical Medical Ethics (GCA), Department of Health Studies (LPC), Biological Sciences Collegiate Division (DM), and Harris School of Public Policy (DOM), University of Chicago, Chicago, Ill; Pacific Health Research Institute (CWT); and Department of Family Practice and Community Health (CWT), John A. Burns School of Medicine, University of Hawaii, Honolulu, Hawaii.

Address correspondence and requests for reprints to Dr. Alexander: RWJ Clinical Scholars Program, The University of Chicago, 5841 S. Maryland, MC 2007, Chicago, IL 60637 (e-mail: galexand@uchicago.edu). out-of-pocket costs are a persistent concern among the public. ${ }^{3}$ Furthermore, these costs are associated with medication nonadherence and may impede physicians' efforts to provide quality care. ${ }^{4-6}$

Previously, we examined the beliefs and experiences of a group of general internists and their patients regarding the discussion of out-of-pocket costs. ${ }^{7}$ We found that one third of patients reported a burden from these costs. In addition, $63 \%$ of patients reported a desire to discuss these costs and $79 \%$ of physicians believed that patients in general want to discuss out-of-pocket costs. By contrast, only $35 \%$ of physicians and $15 \%$ of patients reported ever having discussed the study patients' out-of-pocket costs. In this article, we describe the reported barriers to discussing outof-pocket costs among the subset of patients and physicians who recalled a specific instance when they wanted to discuss out-of-pocket costs but did not do so.

\section{METHODS}

\section{Study Design and Subjects}

Details of our study design have been described previously. ${ }^{7}$ We recruited 3 large academic and 18 smaller community general internal medicine practices in a large midwestern metropolitan area. Potential participants were told the general goal of the study, "to learn about factors doctors and patients consider as they make medical decisions," but were not prospectively told the specific study aims. Trained interviewers attended a morning or afternoon session of each participating physician's practice and invited consecutive patients to complete a short verbal questionnaire after their visit. At the end of the half-day, each participating physician completed a written questionnaire. We included all patients 18 years or older and excluded patients who did not speak English or who were cognitively impaired. The institutional review boards at each participating academic site approved the study protocol and strict measures were undertaken to preserve participant confidentiality.

\section{Surveys}

Based on pilot interviews with physicians, we developed patient and physician questionnaires (available upon request) to examine their beliefs and practices regarding discussions of out-of-pocket costs. Both patient and physician surveys included an item examining whether there had ever been a specific time when they wanted to discuss out-of-pocket costs (with any physician/patient on any visit) but did not do so. The surveys included an open-ended 
question requesting respondents who answered affirmatively to state the reasons why they did not discuss out-of-pocket costs when they wished to do so. Because this query was based on actual recollection of a prior clinical experience rather than on more general beliefs or preferences, the overall proportion of study subjects reporting barriers was smaller than that reporting a preference to discuss out-of-pocket costs. Surveys also included three items examining whether patients were burdened by out-of-pocket costs. Patients were considered to be burdened if they reported cost-related medication nonadherence during the previous 12 months, problems paying medical bills, or agreed or strongly agreed that they had a subjective burden from their out-of-pocket costs. Surveys were extensively piloted and revised prior to administration from March through November 2002.

\section{Analysis}

We used descriptive statistics to examine our primary outcome variable which was defined as the proportion of patients and physicians who recalled a specific time when they wanted to discuss out-of-pocket costs but did not do so. We then examined the bivariate and multivariate associations between patient (age, gender, race, education, selfreported health, number of comorbid conditions, number of prescription medicines, self-reported burden, presence of prescription coverage, length of relationship with physician, number of physician visits in previous 6 months), physician (gender, length since graduation), and practice (academic vs community) characteristics and this outcome variable. In the regression model, we included predictor variables that were of borderline significance on bivariate analysis $(P<.10)$, contained key sociodemographic information, or in which we had a substantive interest. We excluded two predictor variables (number of comorbid conditions and self-reported health) due to covariance with number of prescription medicines ( $r=.43$ to $.65 ; P<.0001)$. Because our primary outcome was common $(>10 \%)$, we derived prevalence (risk) ratios (PRs) from the odds ratios (ORs) to better estimate the true associations between our predictor variables and the outcome variable of interest. ${ }^{8}$

Respondents' self-reported barriers to discussing costs were then transcribed verbatim and initially examined and categorized into themes by one of the investigators (GCA). These categories were then reviewed and revised for clarity and breadth. Three investigators then independently coded each of the responses (GCA, DM, and LPC). Disagreement between coders about the coding of any of the responses was resolved by consensus among these members of the research team.

\section{RESULTS}

\section{Recruitment and Subject Characteristics}

Approximately $80 \%$ of patients approached participated in the study. Among the 484 participating patients,
Table 1. Patient Characteristics $(N=484)^{*}$

\begin{tabular}{ll}
\hline \hline Mean age, y (range) & 54 (18 to 94) \\
Female, \% & 64 \\
Ethnicity, \% & \\
African American & 42 \\
White & 42 \\
Other & 17 \\
Chronic conditions, \% & 18 \\
None & 30 \\
1 & 19 \\
2 & 33 \\
3+ & \\
Education, \% & 26 \\
High school or less & 27 \\
Some college & 28 \\
College degree & 20 \\
Professional or graduate degree & \\
Self-reported insurance status, \% & 68 \\
Private or employer purchased & 4 \\
Medicare with supplemental & 18 \\
Medicare without supplemental & 11 \\
Medicaid or other & $<1$ \\
Uninsured & \\
Practice setting, \% & 63 \\
University-based academic practice & 37 \\
Community-based practice & 90 \\
Have at least partial prescription drug & \\
coverage, \% & \\
\hline
\end{tabular}

* Column percentages may not equal $100 \%$ due to rounding.

the mean age was 54 years (range 18 to 94), 64\% were female, $42 \%$ were African American, $68 \%$ had private or employer-purchased insurance, and 90\% had at least partial prescription drug coverage (Table 1). Only 1 patient was uninsured. Of the 133 participating physicians, the median length in practice was 8 years (range 1 to 55), 55\% were male, and 55\% worked in an academic setting.

\section{Characteristics of Participants Reporting Barriers to Discussions}

Overall, 54 (11\%) of 484 patients and 27 (20\%) of 133 general internists were able to recall a specific instance when they wanted to discuss out-of-pocket costs but did not do so. In bivariate analyses, patients who reported missed opportunities to discuss out-of-pocket costs were more likely to be burdened by their out-of-pocket costs (80\% vs $20 \% ; P<.001$ ), nonwhite ( $16 \%$ vs $5 \% ; P<.001$ ), and without a college education $(24 \%$ vs $8 \%$; $P<.01)$. However, there were no statistically significant associations between physician or practice characteristics examined (e.g., gender, length since graduation, academic vs community practice) and patients' reports of wanting to discuss outof-pocket costs but not doing so. In multivariate analysis controlling for patient age, gender, race, education, number of medicines, and global burden from out-of-pocket costs, patients who were burdened by their out-of-pocket costs (PR, 7.21; 95\% confidence interval [CI], 4.13 to 11.55 ) and nonwhite (PR, 2.10; CI, 1.23 to 3.18 ) were more likely to 
Table 2. Patient and Physician Barriers ( $N=54$ Patients and 27 Physicians)

Patient Barriers

Discomfort

Insufficient time

Didn't know out-ofpocket costs would

be a problem

Fear of compromised quality

Unknown physician role

Other $^{\dagger}$

Physician Barriers

Time

Physician lacked solution

Discomfort

Fear of compromised quality

MD lacked knowledge

Other

\section{Illustrative Examples}

"I didn't feel comfortable bringing up the issue.... I didn't know if it was normal"

"Personal embarrassment about talking about money with other people"

"They're too busy"

"Because from past experience it's not considered appropriate due to time constraints..."

"I thought that he wasn't a part of it or that it wouldn't be helpful"

"I didn't know if it would make a difference"

"Because I didn't know if my prescription was covered by my insurance"

"At the time, I didn't have a lot of bills. I didn't think it was necessary"

"For fear that they would give me a substitute that wouldn't work as well"

"It might jeopardize my health"

"Because you're not sure if he has anything to do with it"

"Because from past experience it's not considered appropriate due to...role issues"

"Some of them may not know what the costs are"

"Slipped my memory"

"She hears it all day long"

"Time limitation; too many other issues"

"Because I had no solution to offer"

"Sometimes I feel like it is better for me not to know the patient's burden because it makes me feel bad that they can't afford the medicine"

"Unsure if it was appropriate to ask what their insurance covered as it implies treatment might vary based on method of payment"

"I don't know each plan's co-insurance"

"My own lack of knowledge of insurance and issues of coverage"

"Mix of patients-poor and wealthy...answers will depend"
Frequency, $\% *$

9

* Column totals may be more than 100\% due to multiple barriers cited by some respondents.

† "Other" category for patients includes: forgot to mention, first time seeing physician, someone other than the respondent discussed out-ofpocket costs with the physician, and no or unclear reason provided.

recall a specific time when they wanted to discuss out-ofpocket costs but did not do so than were their counterparts. There were no significant associations between physician gender, length since graduation, or practice location (community vs academic practice) and physicians' likelihood of recalling a time when they wanted to discuss out-of-pocket costs but did not do so.

\section{Barriers to Discussions}

Table 2 includes the barriers identified by patients and physicians, as well as illustrative quotes taken verbatim from respondents' comments. Patients reported a broad range of barriers to discussing out-of-pocket costs with their physician. These barriers included their own discomfort discussing out-of-pocket costs (19\%), insufficient time (13\%), a belief that the physician did not have a viable solution to offer ( $11 \%$ ), and concerns regarding the impact of discussions on their quality of care (9\%). Among physicians, the most common barriers reported were insufficient time (67\%), a belief that they did not have a solution to offer (19\%), discomfort (11\%), and fear of compromised quality of care $(8 \%)$.

\section{DISCUSSION}

In this study, 1 in 10 patients and 1 in 5 physicians recalled a specific instance when they wanted to discuss out-of-pocket costs but did not do so. Previous research suggests that even among patients and physicians who believe that discussions of out-of-pocket costs are important, these discussions occur infrequently. ${ }^{7}$ Poor physician knowledge of prescription costs may be one reason for this finding. ${ }^{9,10}$ Our research suggests additional barriers that may prevent greater communication about out-of-pocket costs and it provides insight from both patients' and physicians' perspectives. It suggests that interventions to promote communication between patients and physicians about out-of-pocket costs should focus on barriers perceived by both parties, including patient and physician discomfort, the perceived absence of viable solutions, and insufficient time.

\section{Discomfort}

There are several reasons why both patients and physicians may be uncomfortable discussing out-of-pocket costs. Physicians receive little, if any, training in how to help patients navigate the cost-quality trade-offs that are so prevalent in clinical medicine. ${ }^{11}$ Patients, on the other hand, may be embarrassed about discussing their personal finances with their physician and may not be sure if doing so is common or appropriate. In addition, some patients and physicians expressed concern regarding how considerations of cost might compromise the quality of care 
patients receive. Although we did not examine the basis for these concerns in detail, possible contributors include misconceptions about differences between brand name and generic drugs, a belief that more expensive treatments are always of higher quality than less expensive ones, and decreased social trust that health care institutions have patients' best interests in mind. Because discussing outof-pocket costs may cause discomfort or be a sensitive topic for some, physicians may be best able to raise the discussion if they do so regularly and preface any questions with a statement to help decrease the likelihood of patients denying any burden from their costs due to their own embarrassment or shame. Possible language physicians may use to broach the subject includes: "Many patients have a hard time affording the cost of their medicines.... Do you have any problem with this?" or "By the way, I know that medications can be expensive.... How are you doing with the cost of your medications?"

\section{Absence of Viable Solutions}

Although we examined out-of-pocket costs for all types of health care, we included several items focusing specifically on out-of-pocket prescription costs. We focused on these costs because they account for almost half of overall expenditures for prescription medicines, affect a large sector of the population, and are the focus of ongoing debates regarding a national Medicare prescription drug benefit. ${ }^{1,12}$ Furthermore, research suggests that high out-of-pocket costs for prescription medicines may compromise patients' care. $^{4-6}$ As depicted in Table 3 , a variety of methods have been suggested to physicians as a means to reduce patients' out-of-pocket prescription costs. ${ }^{13-17}$ Nevertheless, a considerable proportion of both patients and physicians felt that physicians cannot offer a viable solution to patients' burden. Further work is needed to establish how effective the interventions described in Table 3 can be in reducing patients' out-of-pocket costs without compromising their quality of care. Table 3. Interventions Physician May Use to Assist Patients
Burdened by Their Out-of-pocket Prescription Costs

Switch from brand name to generic drug

Switch to a cheaper brand name drug within the same drug class

Switch from nonformulary to formulary drug

Critically review medication list and consider selective discontinuation of nonessential drugs

Prescribe a higher dose of the medicine and ask the patient to split the pills

Refer patient to a pharmaceutical company assistance program

Refer patient to a public aid agency or social worker

Give the patient office samples

Recommend the use of an over-the-counter medicine as a substitute

\section{Insufficient Time}

Not surprisingly, many physicians mentioned time pressures as the primary barrier preventing discussions regarding out-of-pocket costs. One strategy to promote communication about out-of-pocket costs is to educate physicians to identify patients who have experienced cost-related medication nonadherence or are otherwise burdened from their out-of-pocket costs. ${ }^{4}$ However, predictors of cost-related medication nonadherence have been developed at a population level and it is unknown how effectively physicians can identify individual patients burdened by their out-of-pocket costs. An alternative, and potentially complementary, strategy to promote patientphysician communication about out-of-pocket costs is to encourage patients to raise their concerns when present. A widespread campaign conducted by the American Association of Retired Persons (AARP) utilized national media to promote such dialog ${ }^{18}$; however, the effectiveness of this campaign as well as social marketing techniques on a physician or practice level have not been established. Regardless of whether physicians, patients, or both are targeted, efforts to encourage communication about costs may be most effective and actionable at the point of test or treatment ordering, such as when the physician is writing or renewing a prescription. Furthermore, nonphysician members of the medical team, including nurses, social workers, and pharmacists, may be important allies in efforts to assist patients burdened by high out-of-pocket costs. ${ }^{17}$

\section{Limitations}

Our study had several limitations. First, we examined a group of general internists and their patients in one metropolitan area of the country, and our examination of barriers was limited to the subset of patients and physicians able to recall a specific instance when they wanted to discuss out-of-pocket costs but did not do so. Second, our findings are based on self-report and it is possible that one barrier may be perceived as more important than it actually is (e.g., insufficient time being reported as a proxy for discomfort with the issue). Finally, although we identified several common barriers to patient-physician discussion about out-ofpocket costs, our cross-sectional study design is unable to discern the impact that overcoming these barriers may have on patient satisfaction, health care utilization, or outcomes.

\section{CONCLUSION}

Rising out-of-pocket costs, combined with evidence that for some patients these costs may compromise quality of care, challenge patients and physicians to discuss these costs and the cost-quality trade-offs that they necessitate. Efforts to promote such communication should focus on brief, actionable alternatives that physicians can undertake to advocate maximally on their patients' behalf. Further work is needed to examine the impact of more frequent 
patient-physician communication about out-of-pocket costs. It is plausible that such discussions may lead physicians to critically review patients' medications and identify medicines that can be safely discontinued or changed. Furthermore, independent of changes in patients' prescriptions, such discussions may enhance patient satisfaction, improve adherence, and fortify the physician-patient relationship. These changes could plausibly improve quality and lower costs of care.

This work was supported by funding from the Robert Wood Johnson Foundation Clinical Scholars Program. The content of this publication does not necessarily reflect the views or policies of the Robert Wood Johnson Foundation.

The authors gratefully acknowledge the participating patients and physician practices, Alison Filauro, Jeffery Greggs, Diane McFadden, and Tejal Shah for their role as study interviewers, and Jim Foody, Alex Lickerman, Marjorie McLaughlin, Terry Hammonds, and the Medical Group Management Association for assistance with practice, physician, and patient recruitment.

Participating practices: Advocate Health Center Logan Square; Antillas Family Medical Center; Chicago Family Health Center; Commonwealth Medical Associates; Founders Medical Group; Hyde Park Associates in Medicine; Internal Medicine Associates; James H. Cohn, MD; Northwestern Medical Faculty Foundation General Internal Medicine; Robert Holstein, MD; Rush University Internists; Sage Medical Group; Drs. Tatar, Tatar, Buchanan, Hunt, and Sun; University of Chicago Primary Care Group; University of Chicago Physicians Group Stony Island; University of Chicago Physicians Group E. 47th Street; Westcare Medical Center; West Suburban Hospital Internal Medicine Center; WholeHealth Chicago.

\section{REFERENCES}

1. Medical Expenditure Panel Survey Highlights. Distribution of Healthcare Expenses, 1996. May 2000. Rockville, MD: Agency for Healthcare Research and Quality. Pub. No. 00-0024.
2. Levit K, Smith C, Cowan C, Lazenby H, Sensenig A, Catlin A. Trends in U.S. health care spending, 2001. Health Aff. 2003;22:154-64.

3. Donelan K, Blendon RJ, Schoen C, Davis K, Binns K. The cost of health system change: public discontent in five nations. Health Aff. 1999;18:206-16.

4. Steinman MA, Sands LP, Covinsky KE. Self-restriction of medications due to cost in seniors without prescription coverage. J Gen Intern Med. 2001;16:793-9.

5. Rector TS. Exhaustion of drug benefits and disenrollment of Medicare beneficiaries from managed care organizations. JAMA. 2000;283:2163-7.

6. Federman AD, Adams AS, Ross-Degnan D, Soumerai SB, Ayanian JZ. Supplemental insurance and use of effective cardiovascular drugs among elderly Medicare beneficiaries with coronary heart disease. JAMA. 2001;286:1732-9.

7. Alexander GC, Casalino LP, Meltzer DO. Patient-physician communication about out-of-pocket costs. JAMA. 2003;290:953-8.

8. Zhang J, Yu KF. What's the relative risk? A method of correcting the odds ratio in cohort studies of common outcomes. JAMA. 1998;280:1690-1.

9. Glickman L, Bruce EA, Caro FG, Avorn J. Physicians' knowledge of drug costs for the elderly. J Am Geriatr Soc. 1994;42:992-6.

10. Reichert S, Simon T, Halm EA. Physicians' attitudes about prescribing and knowledge of the costs of common medications. Arch Intern Med. 2000;160:2799-803.

11. Ubel PA. Pricing Life: Why It's Time for Health Care Rationing. Cambridge, Mass: The MIT Press; 2000.

12. Iglehart JK. Medicare and prescription drugs. N Engl J Med. 2001;344:1010-5.

13 Chisholm MA, DiPiro JT. Pharmaceutical manufacturer assistance programs. Arch Intern Med. 2002;162:780-4.

14. Montemayor K. How to help your low-income patients get prescription drugs. Fam Pract Manag. 2002;9:51-6.

15. Jacob J. Pressure mounts to prescribe more generics. AMA News. February 18, 2002:21 (column 1).

16. Stafford RS, Radley DC. The potential of pill splitting to achieve cost savings. Am J Manag Care. 2002;8:706-12.

17. Prutting SM, Cerveny JD, MacFarlane LL, Wiley MK. An interdisciplinary effort to help patients with limited prescription drug benefits afford their medication. South Med J. 1998;91:81520.

18. Stapleton S. AARP campaign attacks rising drug costs. AMA News. May 6, 2002:42 (column 1). 